\title{
Article \\ Classification of Chaotic Signals of the Recurrence Matrix Using a Convolutional Neural Network and Verification through the Lyapunov Exponent
}

\author{
Jaehyeon Nam and Jaeyoung Kang * \\ Department of Mechanical Engineering, Inha University, Incheon 22212, Korea; jaehyeon@inha.edu \\ * Correspondence: kangj@inha.ac.kr
}

\begin{abstract}
This study classified chaotic time series data, including smooth and nonsmooth problems in a dynamic system, using a convolutional neural network $(\mathrm{CNN})$ and verified it through the Lyapunov exponent. For this, the classical nonlinear differential equation by the Lorenz model was used to analyze a smooth dynamic system. The vibro-impact model was used for the nonsmooth dynamic system. Recurrence is a fundamental property of a dynamic system, and a recurrence plot is a representative method to visualize the recurrence characteristics of reconstructed phase space. Therefore, this study calculated the Lyapunov exponent by parametric analysis and visualized the corresponding recurrence matrix to show the dynamic characteristics as an image. In addition, the dynamic characteristics were classified using the proposed CNN model. The proposed CNN model determined chaos with an accuracy of more than $92 \%$.
\end{abstract}

Keywords: convolutional neural network; Lyapunov exponent; recurrence plot; chaos

Citation: Nam, J.; Kang, J. Classification of Chaotic Signals of the Recurrence Matrix Using a Convolutional Neural Network and Verification through the Lyapunov Exponent. Appl. Sci. 2021, 11, 77. https://dx.doi.org/10.3390/ app11010077

Received: 30 November 2020 Accepted: 18 December 2020 Published: 24 December 2020

Publisher's Note: MDPI stays neutral with regard to jurisdictional claims in published maps and institutional affiliations.

Copyright: () 2020 by the authors. Licensee MDPI, Basel, Switzerland. This article is an open access article distributed under the terms and conditions of the Creative Commons Attribution (CC BY) license (https: / / creativecommons.org/ licenses/by/4.0/).

\section{Introduction}

The problem of predicting chaotic dynamics has been studied for a long time in dynamical systems [1-5]. Chaos is a non-periodic solution with unstable parameters in a dynamic system, but it has a limitation within a strange attractor. This has been introduced into widely known nonlinear equations, such as Lorenz, Rossler [1], and Van der Pol. The classical method to analyze a dynamic system uses a time series and a phase portrait. On the other hand, after the Cooley-Tudkey algorithm in the 1960s, research and development of many dynamic systems have led to studies, such as higher-order spectrum analysis (HOSA), logistic maps, the Lyapunov exponent, and recurrence plots to analyze methods, including high-dimensional nonlinearity. Among them, the Lyapunov exponent has been developed and studied in many papers as the most popular method for quantifying chaos. Wolf et al. [6] developed a numerical algorithm determining the Largest Lyapunov exponent. On the other hand, this algorithm cannot be applied to dynamic systems including discontinuities, and can only be used in smooth dynamic systems. Muller's algorithm, developed to overcome this problem, can be used in a nonsmooth dynamical system, making it possible to calculate the Lyapunov exponent by applying it to impact and friction problems, including discontinuities [7].

In mechanical vibration, chaotic signals are a phenomenon that occurs in various applications, such as friction-induced vibration, impact noise, and fault diagnosis. In particular, mechanical vibrations can generate a chaotic phenomenon because they contain strong nonlinearity stemming from the discontinuity of friction and impact. Vibration problems occurring in general friction systems, including nonlinearity, were analyzed for stability via linearization [8-10]. On the other hand, after frictional vibration occurs, the nonlinear signal of the vibration increases and can become chaotic. Kang [11] examined the chaos phenomenon caused by friction-induced vibration using a 2-DOF model that includes the negative slope of a continuous friction curve. In particular, the negative slope was found to 
be a control parameter capable of expressing chaotic characteristics. In addition, studies on the parameters that express the chaotic characteristics occurring in dynamic systems, including friction, have been investigated in many papers [2-5].

In the impact condition, the collision force is extremely discontinuous at the moment of impact. The resulting kinetic phenomena for impact motion have been studied extensively to assess the chaos phenomenon. Serweta et al. [12,13] investigated the chaotic characteristics by calculating the Lyapunov exponent for a dynamic system with discontinuities through Muller's procedure for Hertz's and Newton's contact models. Kang [14] calculated the Lyapunov exponent for the truncated number of modes of the impact beam under distributed contact using the continuous beam model.

In addition to the theoretical approach, the vibration signal can be analyzed using the visualization method of the time series. The analysis method of visualization of the vibration signals, including high-order nonlinearity, was developed in studies, such as Gauss wavelets $[15,16]$ and recurrence plots [17]. Marwan et al. [17] introduced various recurrence plot analyses as a visualization method of dynamic characteristics occurring in a complex system and analyzed the dynamic characteristics. The phase space is reconstructed using the time delay method. The embedding dimension and time delay must be determined to express the reconstructed phase space by the time delay method. The time delay method is a dynamic problem that can be determined using the Mutual information method [18]. The embedding dimension is a geometric problem that is being studied to obtain the minimum physical embedding dimension $[18,19]$. In this study, the embedding dimension was determined using the method of false nearest neighbors (FNN) proposed by Kennel. Despite the many studies to understand the characteristics of dynamic systems, the decision on the visualized dynamical characteristics was made by the subjective judgment of the engineer. Therefore, uncertainty is always present, and a dynamical property cannot be judged without a trained engineer.

As studies of visualization of time series have been carried out, methods of distinguishing the characteristics of dynamic signals through the procedure of quantifying recurrence characteristics have been proposed. In particular, recurrence quantification analysis (RQA) can determine recurrence patterns through indices expressed as such in the recurrence rate (RR), the determinism (DET), and the average diagonal line length [3,20-22]. Cluster numbers provided the visualization of the bifurcation [21]. However, these methods for quantifying recurrence patterns provide indices as a reference, and a professional engineer is required to reinterpret these numbers. On the contrary, the Lyapunov exponent is known as the most deterministic tool to determine chaos when the governing equation is explicitly known [20].

In the recent rapid development of artificial intelligence, however, many algorithms using machine learning have been developed. In particular, image classification developed numerous algorithms based on a convolutional neural network. In 2015, ResNet in the algorithm proposed by He et al. [23] exceeded the human cognitive ability in image classification. A network adopting the Residual Learning method has an error rate of $4 \%$, which is lower than the error of $5 \%$ of human classification ability and has improved learning ability over the existing learning speed. Based on the above results, this paper proposes a convolutional neural network (CNN) model that can classify chaos by machine learning. The model verified the appropriateness of the proposed CNN model by calculating the Lyapunov exponent. Therefore, the present method is proposed in order to more quickly and accurately determine chaos characteristics of general complex signals after learning the predetermined data using the Lyapunov exponent of explicitly known analytical equations.

\section{Methods}

Figure 1 shows the vibro-impact model with mass, linear spring, and nonlinear elastic contact. The spring $k$ and $k_{e x}$ are linear spring coefficients, and the system can be under external kinematic excitation forcing. The spring $k_{e x}$ at the left end has an amplitude $f_{0}$ and an excitation frequency $\omega_{e x}$, and is excited harmonically. The distance between the impact 
surface at the static equilibrium position of the system is $r$. In addition, the nonlinear elastic model of the collision force was defined as Hertz's contact model [12]. The dynamic behavior of the system can be made dimensionless for the generalization and efficiency of interpretation, as shown in Figure 1b.

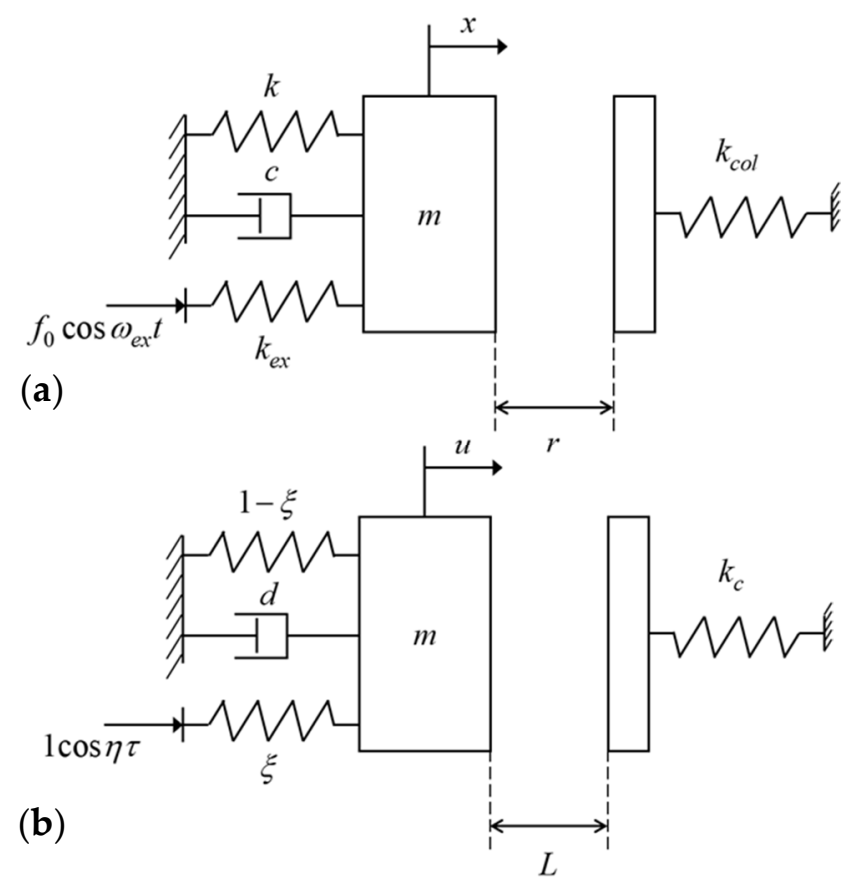

Figure 1. Dynamical impact system with external excitation, (a) 1-D impacting oscillator, (b) dimensionless form of (a).

Consider a mass $\mathrm{m}$ attached to a spring stiffness coefficient $k, k_{e}$ and coefficient of viscous damping $c$. If the impact force is 0 , it is only a single degree-of-freedom equation of motion by excitation. The equation of motion is as follows:

$$
m \ddot{x}+c \dot{x}+\left(k+k_{e x}\right) x=f_{0} k_{e x} \cos \omega_{e x} t .
$$

Using the dimensionless time, $\tau=t \sqrt{(k+k e) / m}$ and the coordinate transformation $x(t)=X(t)$, the dimensionless equation of motion can be written as:

$$
X^{\prime \prime}+X^{\prime}+X=\xi f_{0} \cos \eta \tau
$$

where prime is the differentiation with respect to $\tau(\geq 0)$, and $\omega_{n}=\left(\sqrt{\left(k+k_{e}\right) / m}\right)$ is the natural frequency of the undamped system. $\eta=\omega_{e x} / \omega_{n}$ is the relation between the excitation and natural frequency. $d=c / \sqrt{\left(k+k_{e}\right) m}$ is the dimensionless damping ratio, $\xi=k_{e} /\left(k+k_{e}\right)$ is the relation between the spring coefficient, and $u=X / f_{0}$ is the dimensionless displacement related to the magnitude of excitation forcing,

$$
u^{\prime \prime}+u^{\prime}+u=\xi \cos \eta \tau .
$$

The most popular Hertz's model of contact is the nonlinear elastic model, and the dimensionless collision force is given by:

$$
\begin{gathered}
F_{L}=0 \text { if } u<L \\
F_{L}=k_{c}(u-L)^{3 / 2} \text { if } u \geq L
\end{gathered}
$$


where $k_{c}=k_{\text {col }} \sqrt{f_{0}} /\left(k+k_{e}\right)$ is the dimensionless ratio of the stiffness of the surface, which is dependent on the elastic properties and geometry of the rigid body. The dimensionless equation of motion with impact force can be written as:

$$
u^{\prime \prime}+u^{\prime}+u=\xi \cos \eta \tau+F_{L} H(u-L)^{3 / 2}
$$

where $H$ is the Heaviside function. For numerical integration, Equation (4) can be rewritten in the state space such that:

$$
\begin{aligned}
& \dot{z}_{1}=z_{2} \\
& \dot{z}_{2}=-z_{2}-z_{1}+\xi \cos z_{3}+F_{L} H(u-L)^{3 / 2} . \\
& \dot{z}_{3}=\eta
\end{aligned}
$$

The dynamical equation of motion can be converted to the state-space vector form as follows:

$$
\dot{\mathbf{z}}=\mathbf{f}(\mathbf{z}), \mathbf{z}\left(t_{0}\right)=\mathbf{z}_{0} .
$$

Here, $\mathbf{y}\left(t_{0}\right)$ is the initial condition of the perturbed solution, and Equation (7) is taken for the Taylor series at $\mathbf{z}(t)$ such that:

$$
\delta \dot{\mathbf{z}}=\left.\frac{\partial \mathbf{f}}{\partial \mathbf{z}}\right|_{\mathbf{z}=z_{i}} \cdot \delta \mathbf{z}+O_{(2)}, \delta \mathbf{z}\left(t_{0}\right)=\delta \mathbf{z}_{0} .
$$

By letting $\delta \mathbf{z}=\left[\boldsymbol{\Phi}_{t}\left(\mathbf{z}_{0}\right)\right] \delta \mathbf{z}_{0}$ substitute into perturbation Equation (8), the variation equation can be written as:

$$
\left[\dot{\boldsymbol{\Phi}}_{t}\left(\mathbf{z}_{0}\right)\right] \delta \mathbf{z}_{0}=\left[D_{z} \mathbf{f}\right]\left[\boldsymbol{\Phi}_{t}\left(\mathbf{z}_{0}\right)\right] \delta \mathbf{z}_{0},\left[\boldsymbol{\Phi}_{t_{0}}\left(\mathbf{z}_{0}\right)\right] \delta \mathbf{z}_{0}=[\mathbf{I}] \delta \mathbf{z}_{0}
$$

where $\left[D_{z} \mathbf{f}\right],[\mathbf{I}]$, and $\left[\boldsymbol{\Phi}_{t}\left(\mathbf{z}_{0}\right)\right]$ denotes the Jacobian matrix, identity matrix, and solution of the variational equation, respectively. The spectrum of the Lyapunov exponent for the linearized Equation (9) is estimated through Wolf's algorithm using the QR-factorization orthonormalization [6,24].

In this model, Equation (6) represents a nonsmooth dynamical system with discontinuous impact effects. The dynamic system, including the discontinuity at the moment of impact, can be rewritten from Muller's method. The discontinuity occurs at the point of impact $\left(t=t_{i}\right)$.

$$
\begin{array}{r}
t_{i-1}<t<t_{i}: \dot{\mathbf{z}}=\mathbf{f}_{i}(\mathbf{z}), \mathbf{z}\left(t_{i-1}\right)=\mathbf{z}\left(t_{i-1}^{+}\right) \\
t=t_{i}: 0=\mathbf{h}\left(\mathbf{z}\left(t_{i}^{-}\right)\right) \\
\mathbf{z}\left(t_{i}^{+}\right)=\mathbf{g}\left(\mathbf{z}\left(t_{i}^{-}\right)\right) \\
t_{i}<t<t_{i+1}: \dot{\mathbf{z}}=\mathbf{f}_{i+1}(\mathbf{z}), \mathbf{z}\left(t_{i}\right)=\mathbf{z}\left(t_{i}^{+}\right)
\end{array}
$$

The perturbed trajectory is given by:

$$
\begin{gathered}
\tilde{\mathbf{z}}(t)=\mathbf{z}(t)+\delta \mathbf{z}(t) \\
\widetilde{t}_{i}=t_{i}+\delta t_{i}
\end{gathered}
$$

and the perturbed trajectory satisfies the following equation.

$$
\begin{gathered}
\widetilde{t}_{i-1}<t<\widetilde{t}_{i}: \dot{\tilde{\mathbf{z}}}=\mathbf{f}_{i}(\tilde{\mathbf{z}}), \tilde{\mathbf{z}}\left(\widetilde{t}_{i-1}\right)=\tilde{\mathbf{z}}\left(\widetilde{t}_{i-1}^{+}\right) \\
t=\widetilde{t}_{i}: 0=\mathbf{h}\left(\tilde{\mathbf{z}}\left(\widetilde{t}_{i}^{-}\right)\right) \\
\tilde{\mathbf{z}}\left(\widetilde{t}_{i}^{+}\right)=\mathbf{g}\left(\tilde{\mathbf{z}}\left(\widetilde{t}_{i}^{-}\right)\right)
\end{gathered}
$$




$$
\widetilde{t}_{i}<t<\widetilde{t}_{i+1}: \dot{\tilde{\mathbf{z}}}=\mathbf{f}_{i+1}(\tilde{\mathbf{z}}), \tilde{\mathbf{z}}\left(\widetilde{t}_{i}\right)=\tilde{\mathbf{z}}\left(\widetilde{t}_{i}^{+}\right)
$$

where each interval of discontinuities is smooth, and $\mathbf{h}(\mathbf{z})$ and $\mathbf{g}(\mathbf{z})$ are the indicator function and the transition condition, respectively. The plus and minus denote the rightand left-sided limits, and

$$
\begin{gathered}
\delta t_{i}=\widetilde{t}_{i}-t_{i}=-\frac{D \mathbf{h}\left(\mathbf{z}_{i}^{-}\right) \delta \mathbf{z}_{i}^{-}}{D \mathbf{h}\left(\mathbf{z}_{i}^{-}\right) \mathbf{f}_{i}\left(\mathbf{z}_{i}^{-}\right)} \\
\delta \mathbf{z}_{i}^{+}=D \mathbf{g}\left(\mathbf{z}_{i}^{-}\right) \delta \mathbf{z}_{i}^{-}+\left[D \mathbf{g}\left(\mathbf{z}_{i}^{-}\right) \mathbf{f}_{i}\left(\mathbf{z}_{i}^{-}\right)-\mathbf{f}_{i+1}\left(\mathbf{z}_{i}^{+}\right)\right] \delta t_{i}
\end{gathered}
$$

in which

$$
D \mathbf{h}\left(\mathbf{z}_{i}^{-}\right)=\left.\frac{\partial \mathbf{h}(\mathbf{z})}{\partial \mathbf{z}}\right|_{\mathbf{z}=\mathbf{z}_{i}^{-}}, D \mathbf{g}\left(\mathbf{z}_{i}^{-}\right)=\left.\frac{\partial \mathbf{g}(\mathbf{z})}{\partial \mathbf{z}}\right|_{\mathbf{z}=\mathbf{z}_{i}^{-}}
$$

are the Jacobian matrix of the indicator function and transition condition at point $\mathbf{z}_{i}^{-}$, respectively, and $\mathbf{z}_{i}^{-}=\mathbf{z}\left(t_{i}^{-}\right)$and $\mathbf{z}_{i}^{+}=\mathbf{z}\left(t_{i}^{+}\right)$. For the impact oscillator with Hertz's model of contact, the Jacobian matrix of transition condition and indicator function becomes the following matrix:

$$
D \mathbf{h}\left(\mathbf{z}_{i}^{-}\right)=\left[\begin{array}{lll}
1 & 0 & 0
\end{array}\right]^{\mathbf{T}}, D \mathbf{g}\left(\mathbf{z}_{i}^{-}\right)=\mathbf{I} .
$$

The recurrence plot (RP) is used as a tool to visualize the recurrence pattern of a dynamical system. A recurrence matrix is expressed by a series of solution vectors. The corresponding $\mathrm{RP}$ is based on the following recurrence matrix as follows:

$$
\mathbf{R}_{i, j}=\left\|\mathbf{z}_{i}-\mathbf{z}_{j}\right\|, i, j=1, \ldots, N
$$

where $\|\cdot\|$ is an L-2 norm, $N$ is the measured points, and $\left\{\mathbf{z}_{i}\right\}_{i=1}^{N}$ is a system's trajectory in its phase space. Here, the element of phase space indicates the possible state of the system for the time evolution law. In such cases, phase space needs to be reconstructed. The most popular method of reconstruction is the time delay method

$$
\hat{\mathbf{z}}_{i}=\sum_{j=1}^{m} u_{i+(j-1) v} \mathbf{e}_{j}
$$

where $u_{i}=u(i \Delta t)$ is the discrete-time series, $\Delta t$ is the sampling rate, $m$ is the embedding dimension, $v$ is the time delay, and $\mathbf{e}_{j}$ are unit vectors. The reconstruction does not change the dynamical properties, and the reconstructed phase space can be expressed through an appropriately selected embedding dimension and time delay. In general, the time delay can be determined appropriately using the Mutual information method.

During time-delay reconstruction, all self-crossing of the trajectory for the attractor's dimension $d_{A}$ can disappear if it is set to the embedding dimension $d>2 d_{A}$. From a physical point of view, it is very important to determine the minimum embedding dimension to minimize the numerical calculation of the Lyapunov exponent. From Equation (1) in dimension $d, \mathbf{z}_{r}$ is the $r$ th nearest neighbor of $\mathbf{z}$, and the square of the Euclidean distance between the two vectors is

$$
R_{d}^{2}(i, r)=\sum_{e=0}^{v-1}\left[u_{(i+e \tau)}-u_{r,(i+e \tau)}\right]^{2} .
$$

As it expands on dimension $d$ to dimension $d+1$ by time-delay embedding, the Euclidean distance between $r$ th neighbors for dimension $d+1$ can be written as follows:

$$
R_{d+1}^{2}(i, r)=R_{d}^{2}(i, r)+\left[u_{(i+d \tau)}-u_{r,(i+d \tau)}\right]^{2} .
$$


Here, the error for the minimum embedding dimension may be determined through the rate of change of the Euclidean distance as follows:

$$
\sqrt{\frac{R_{d+1}^{2}(i, r)-R_{d}^{2}(i, r)}{R_{d+1}^{2}(i, r)}}>R_{t o l}
$$

where $R_{t o l}$ is the threshold. According to a lecture, false neighbors can be identified clearly if $R_{t o l} \geq 10[18,19]$. Another condition for determining false neighbors is defined based on the fact that the actual value of $R_{d}(i) \equiv R_{d}(i, r=1)$ is similar to the attractor's standard deviation, $R_{A}$, using the finite data onto the noise signal. Thus, the Euclidean distance from the dimension $d+1$ becomes $R_{d+1}(i) \approx 2 R_{A}$. The second criterion for determining false neighbors is as follows:

$$
\frac{R_{d+1}(i)}{R_{A}}>A_{t o l} .
$$
(29).

They are decided as false neighbors based on the conditions of Equations (28) and

\section{Results}

This study aimed to show that it is possible to classify chaos signals and non-chaos signals using a CNN and verify it using the Lyapunov exponent. Figure 2 shows the flow chart of the proposed methodology. First, the nonlinear time series data onto the parametric dynamic system were obtained by numerical analysis using the Runge-Kutta method. Second, the obtained time series data were determined using the FNN algorithm to determine the embedding dimension, and the phase space was reconstructed. Third, the reconstructed data were presented as an unthresholded recurrence plot to visualize the dynamic characteristics. The Lyapunov exponent corresponding to the change in the control parameter was calculated, and it was trained and verified using the proposed CNN model. Table 1 shows the layer type, filter size, and shape of each layer of the proposed CNN model.

\section{Parametric system}

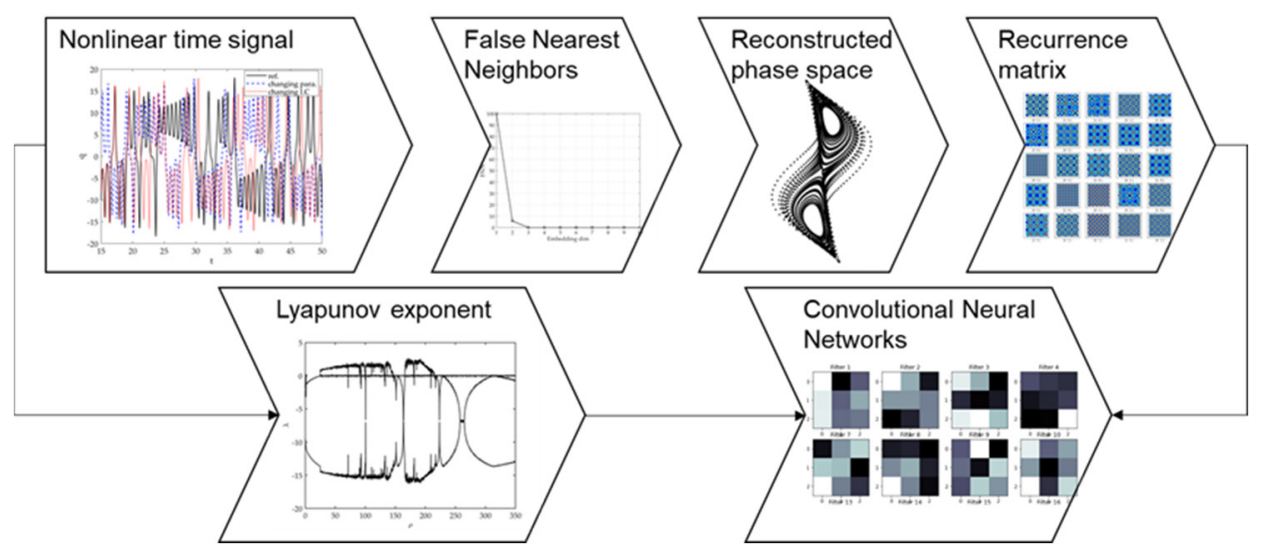

Figure 2. Flow diagram of the proposed methodology.

For image classification using a CNN, many sophisticated models have been developed that transcend human cognitive ability, but the proposed model is simply composed of a two-stage structure to distinguish only chaos and non-chaos, as shown in Figure 3. In each stage, the convolution layer, activation function, and pooling layer were included. The proposed model had two convolution layers with a $32-3 \times 3$ filter and a $64-3 \times 3$ filter, respectively. 
Table 1. CNN model for the Lorenz system.

\begin{tabular}{ccc}
\hline Layer & Output Shape & Param \# \\
\hline Conv2d(Conv2d) & (None, 200, 200, 32) & 896 \\
Batch normalization & (None, 200, 200, 32) & 128 \\
Max pooling2d & (None, 100, 100, 32) & 0 \\
Conv2d 1 & (None, 100, 100, 64) & 18,496 \\
Batch normalization 1 & (None, 100, 100, 64) & 256 \\
Max pooling2d 1 & (None, 50, 50, 64) & 0 \\
Flatten & (None, 160,000) & 0 \\
Dense & (None, 256) & $40,960,256$ \\
Batch normalization 2 & (None, 256) & 1024 \\
Dense 1 & (None, 2) & 514 \\
\hline
\end{tabular}

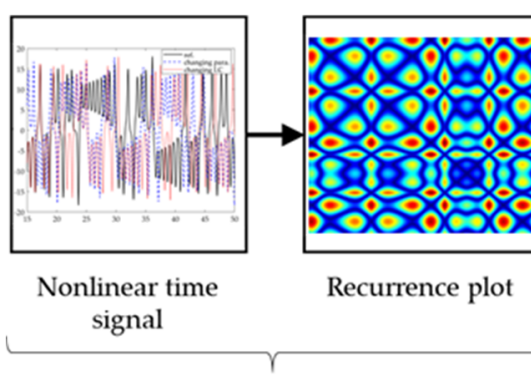

Input

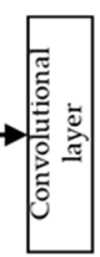

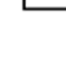

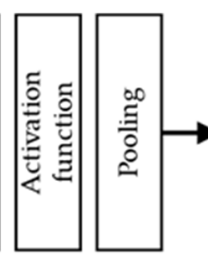

layer

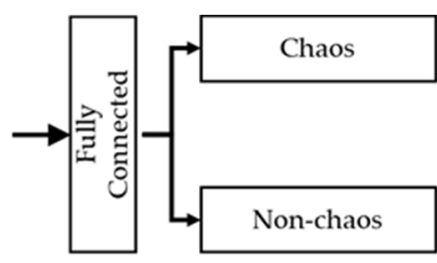

Classification

Figure 3. Flow diagram of the proposed convolutional neural network (CNN) model for the Lorenz system.

As mentioned above, the unthresholded recurrence plot is a tool to visualize the recurrence characteristics of a dynamic system. In the case of chaotic signals, the repetition characteristics can occur in a very short interval. Therefore, the filter size was set as small as possible. In addition, two max pooling layers of $2 \times 2$ were used. At the end of the last stage, through two convolution layers, the feature maps were flattened into a column vector, and the features of the image were classified into a fully connected layer for the two types of signals. Softmax was used as the activation function of the output.

One of the gradient-based optimization methods was used to estimate the minimized cost function of the proposed model. The Adam optimizer is an optimization function based on the gradient decent algorithm and achieved faster convergence [25].

The weight initialization is a very important problem. Many problems can occur if the weight setup is wrong, such as convergence problems and local minimum problems. LeCun initialization follows a Gaussian distribution and uniform distribution of weight initialization for effective backpropagation [26]. Xavier initialization sets the weight depending on the number of previous and next nodes [27]. This is the most generalized method, but the output value showed inefficient results when used in the ReLU function. He initialization was developed to compensate for this [28]. In the proposed model, the weight initialization was performed using the He initialization method following a Gaussian distribution.

The Lorenz system was used as the typical chaotic system for preliminary analysis and $\mathrm{CNN}$ verification. $\rho$ was selected as the control parameter; the other parameters were $\sigma=10$ and $\beta=8 / 3$. The initial conditions were $q(0)=v(0)=0$ and $p(0)=1$. The Lorenz equation is described in the state space such that:

$$
\begin{aligned}
& \dot{q}=-\sigma(q+p) \\
& \dot{p}=q(\rho-v)-p \\
& \dot{v}=q p-\beta v
\end{aligned}
$$


where $\mathbf{q}=\left[\begin{array}{lll}q & p & v\end{array}\right]^{\mathbf{T}}$ is the state vector and $\sigma, \rho$, and $\beta$ are parameters. Figure 4 shows the Lorenz system with chaotic attractors.
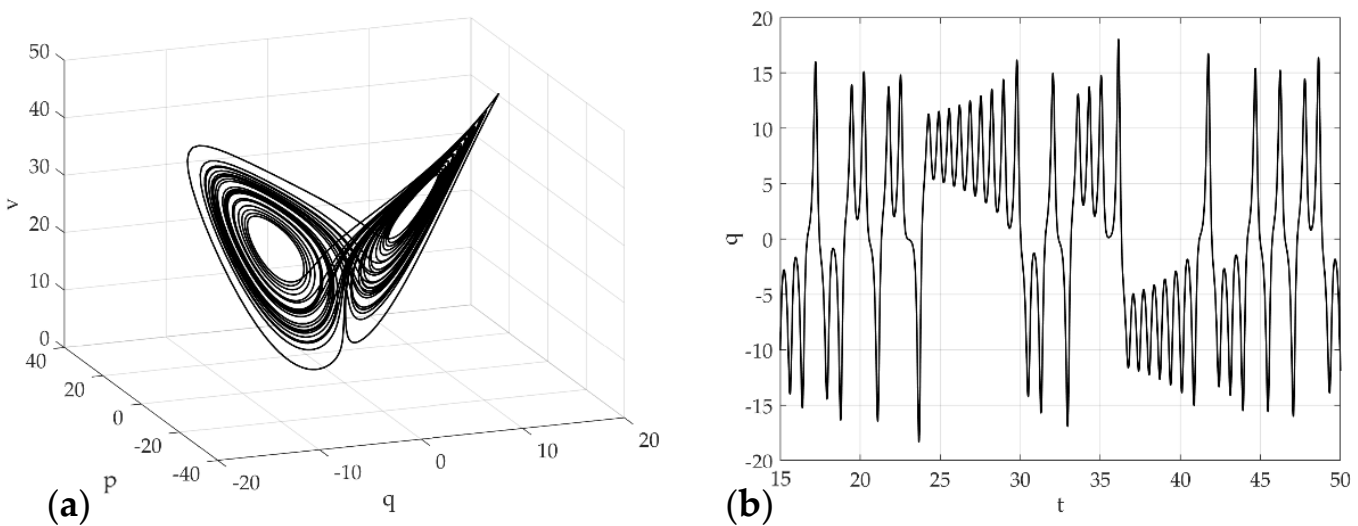

Figure 4. Chaotic attractor for the Lorenz system when $\sigma=10, \beta=8 / 3$, and $\rho=28$; the initial conditions were $q(0)=v(0)=0$ and $p(0)=1,(\mathbf{a})$ Phase portrait, (b) Time series plot of $q(t)$.

In the Lorenz system, infinitesimal differences in the parameters or initial conditions alter the state of the system. As shown in Figure 4a, the trajectory shows a butterfly shape in phase space and moves in an infinite trajectory at a bounded steady state. Figure $4 \mathrm{~b}$ illustrates the time series result of $q$. Figure 5 a presents the Lyapunov exponent of the Lorenz equation with the change in the control parameter. Figure $5 \mathrm{~b}$ shows the unthresholded recurrence plot of visualized chaos and non-chaos. [1 0] is a chaos image and [0 1] is a non-chaos image.

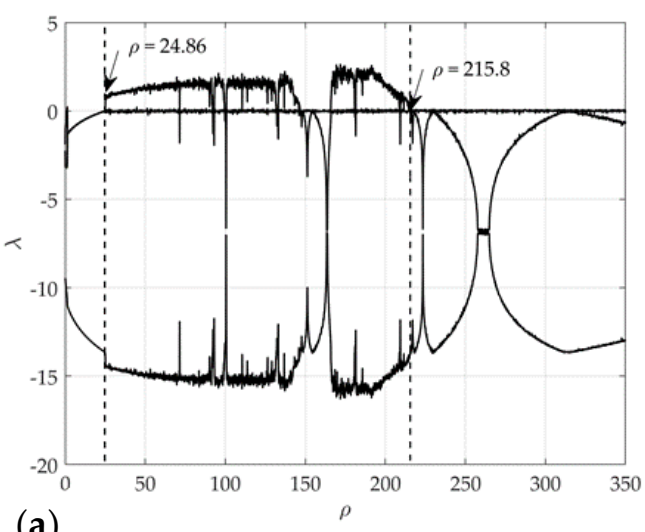

(a)

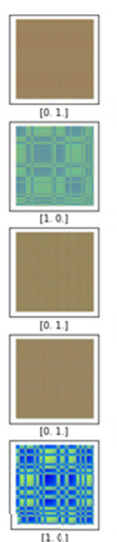

(b)

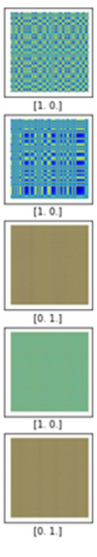

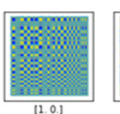
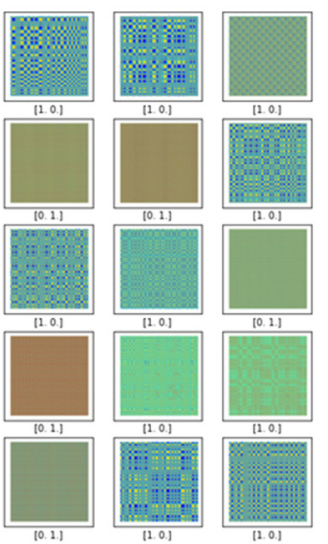
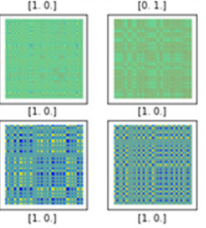

Figure 5. Dataset labeling with respect to the chaotic signal, (a) Lyapunov exponent in the range $0<\rho<350$, (b) recurrence matrix.

The explanation of critical A for the Lyapunov exponent of the Lorenz system has been investigated [6]. To summarize this system briefly, if $215.8>\rho>24.86$, it represents a strange attractor for the control parameter $\rho$ and the initial condition. On the other hand, strange attractors are not displayed under some conditions. On the other hand, if $\rho>215.8$, the system was represented as a stable attractor. In 3D phase space, the Lyapunov exponent has four types of attractors, such as stable fixed points, stable limit cycles, stable two-torus, and strange attractors (Chaos), but only chaos and non-chaos were distinguished in this system. In other words, the unthresholded recurrence matrix can be obtained from the flow of the proposed method in the control parameter representing the chaotic attractor $\left(\max \left(\lambda_{i}\right)>0\right)$ of the dynamic system, which is classified using the CNN. 
The Lorenz system consisted of 3800 datasets and $200 \times 200$ pixels images. The time series analysis and Lyapunov exponents were calculated with a time step of 0.01 and an orthonormalization of 0.1 , respectively. The dataset was divided into three main parts. The total dataset was divided into $70 \%$ of the training dataset and $30 \%$ of the testing dataset. From the divided training dataset, it was divided into $20 \%$ of the validation dataset. The dataset samples used for training are described in Table 2. Because the images are generated sequentially as the Lyapunov exponent increases, errors due to the sequential dataset were removed by shuffling the dataset.

Table 2. Dataset samples.

\begin{tabular}{ccc}
\hline Data & Percentage & Number of Samples \\
\hline Training & $56 \%$ & 2128 \\
Validation & $14 \%$ & 532 \\
Testing & $30 \%$ & 1140 \\
\hline
\end{tabular}

Figure 6 presents the results of the proposed CNN model. The chaos characteristics were trained for 2128 training data samples and simultaneously verified by 532 data samples during each epoch. The tests were then performed on 1140 data samples using the trained CNN model. The batch size was set to 10, and the learning rate of the optimization function was 0.0001. The accuracies and losses were collected as the training and verification data for each epoch, and are plotted in Figure 6a,b. As shown in the learning results, the accuracies were illustrated as a logarithmic function and reached almost $100 \%$. The losses were indicated as a negative exponential function and converge close to $0 \%$. This suggests that the proposed model detects the recurrence properties of the Lorenz system very well.

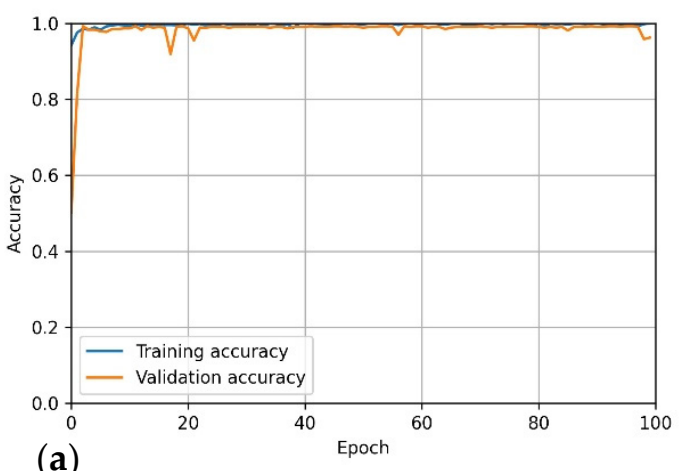

(a)

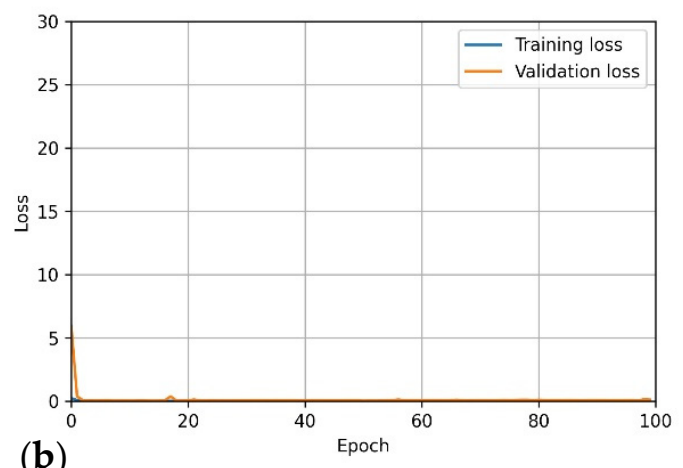

(b)

Figure 6. Results of CNN training for the Lorenz system, (a) accuracy, (b) and loss curves over 100 epochs.

Figure 7 shows the feature map in the first layer. Figure 7a shows the filter map for weight and basis using the He Gaussian method, and Figure $7 \mathrm{~b}$ presents the feature map corresponding to each filter. In Figure $7 b$, the white image means that the feature cannot be found by the corresponding filter. Overall, the filter finds chaos characteristics well. Over 100 epochs, the proposed CNN model classified the chaotic characteristics with $96 \%$ accuracy. This suggests that the proposed CNN model can analyze complex dynamic characteristics that humans cannot recognize with high accuracy. Besides this, the experimental results for 1140 datasets not used for learning were classified with $95 \%$ accuracy. This shows that the $\mathrm{CNN}$ can extract more complex dynamical properties using generalized features from the unthresholded recurrence plot. Therefore, a numerical experiment was performed on the vibro-impact problem, as below. 
(a)
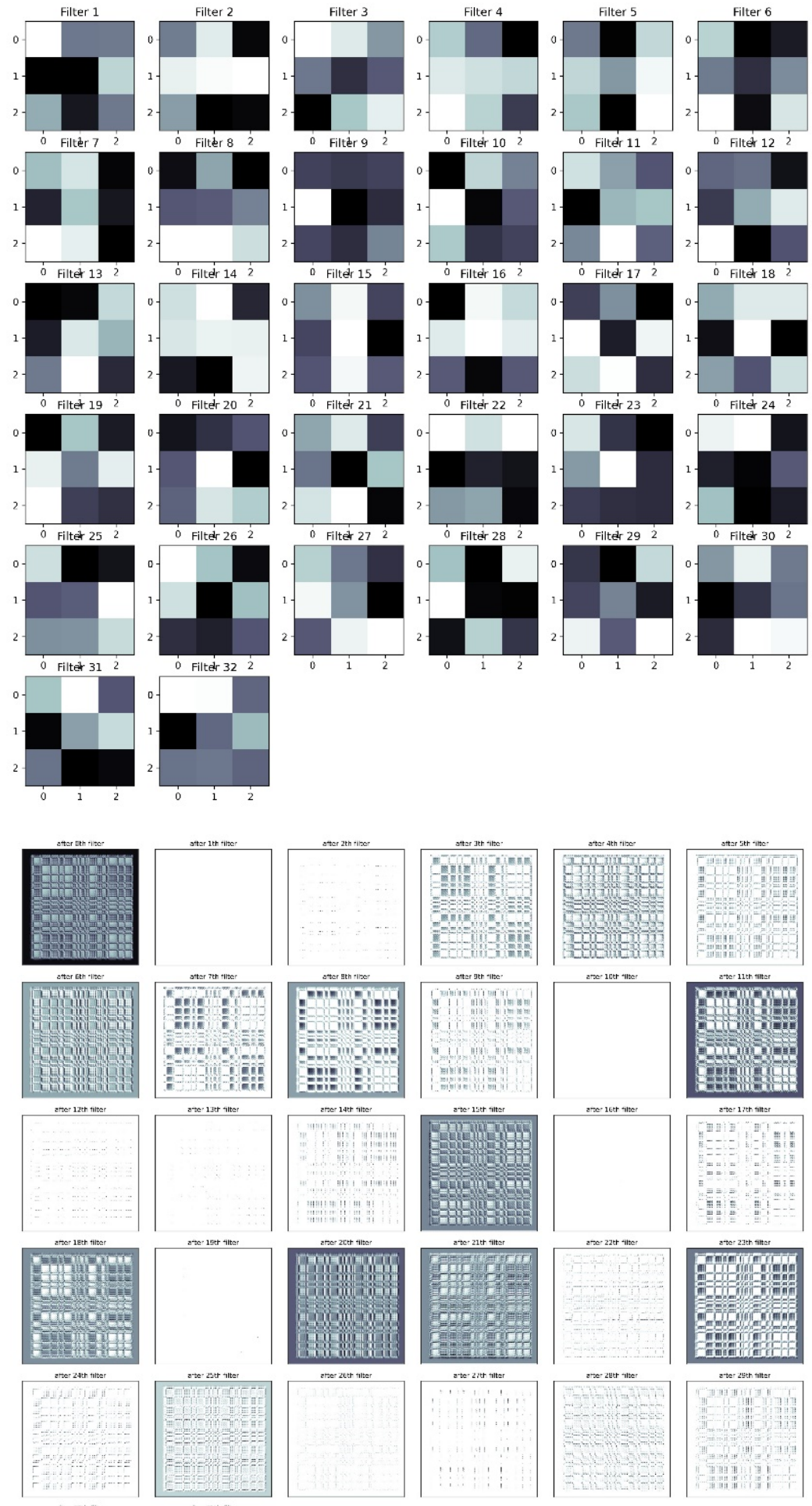

(b)

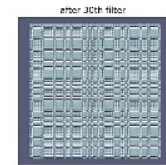

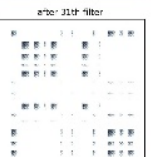

Figure 7. The feature extraction for the first convolution layer, (a) filter map for the first convolution layer, (b) feature map.

In the case of the Lorenz system, it is relatively easy to distinguish the recurrence properties because it is not a system that oscillates for all control parameters. In other words, the system does not vibrate if the Lyapunov exponent reaches a stable equilibrium. Therefore, 
it is not well expressed for the recurrence properties of the dynamical system. On the other hand, the vibration system with discontinuity has a highly complex recurrence property. Therefore, the classification procedure of the proposed methodology was performed by applying Muller's method to Equation (6).

Figure 8 shows the Lyapunov exponent of the vibro-impact model calculated for the control parameter $\eta$; the other parameters and initial conditions were $r=2, \xi=0.05$, $k_{h}=100$, and $\mathbf{z}_{0}=\left[\begin{array}{lll}0.5 & -1.2 & 0\end{array}\right]^{\mathrm{T}}$. In this study, only chaos and non-chaos dynamic characteristics were distinguished for the system with impact and excitation, so other detailed types of attractors were not considered. In the analysis results, the Lyapunov exponent was changed drastically if the control parameter increases as infinitesimal. Figure 9 plots the characteristics of typical attractors for each generated type. Figure $9 a$ and b shows the time series plot and the corresponding phase portrait for the attractor, respectively, for the case $\eta=0.65$. The other cases $\eta=0.7416$ are shown in Figure $9 \mathrm{c}, \mathrm{d}$. The attractor showed a stable limit cycle without impact, and the sign of the largest Lyapunov exponent was $\left(\lambda_{1}, \lambda_{2}, \lambda_{3}\right)=(0,-,-)$, as shown in Figure 9a. If the sign of the largest Lyapunov exponent is zero and the others are negative, it means that the system does not diverge and the oscillation is stable. In other words, it was not made a chaotic trajectory. On the other hand, Figure $9 \mathrm{~b}$ shows that an attractor diverges within the bounded steady state to reach the critical region. A chaotic trajectory different from the periodic or quasi-periodic solution was plotted. Here, the sign of the largest Lyapunov exponent is generated as $\left(\lambda_{1}, \lambda_{2}, \lambda_{3}\right)=(+, 0,-)$. This suggests that it was generated as a strange attractor.

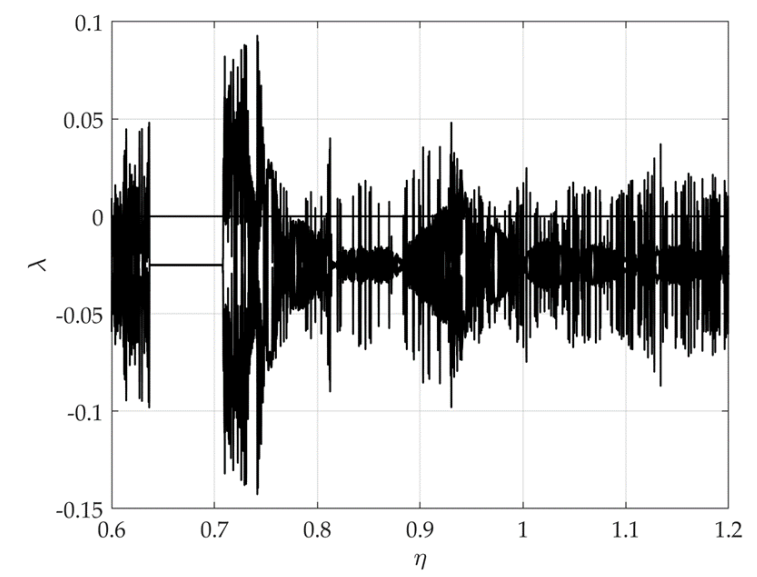

Figure 8. Lyapunov exponents for the impacted vibration system.

Therefore, some of the unthresholded recurrence plots are shown in Figure 10 for classification into two types: $\left(\lambda_{1}, \lambda_{2}, \lambda_{3}\right)=(+, 0,-)$ and others. [1 0 ] and [0 1] below the image represent chaos and non-chaos, respectively. As shown in Figure 10, the images by the chaos system and by the non-chaos system always oscillated. Therefore, images were expressed in a very complex form. The complex systems require more sophisticated neural networks. Therefore, we constructed a neural network using only eight residual blocks based on ResNet, one of the most sophisticated neural networks. In total, 3800 datasets were used for training and consisted of $200 \times 200$ pixels images. The results are shown in Figure 11.

The proposed CNN model was converged with 95\% accuracy over the 200 epochs and appeared in the shape of a logarithmic function. Here, the reason for the fluctuation of accuracy up to the initial 80 epochs was to give a large running rate for rapid convergence of the experiment, and the learning was performed by decreasing the running rate after 80 epochs. On the other hand, the loss was converged over 200 epochs to $0 \%$ in the shape of the negative exponential function. The proposed procedure and method of classification using the CNN model was found on the chaotic characteristics of the vibro-impact model well and classified with high accuracy. As a result of experimenting with 1140 datasets not 
used for training, the chaotic signal was classified with a high accuracy of approximately $92 \%$. This shows that if the characteristics of a relatively very complex dynamic system can be visualized, the characteristics of the signal can be analyzed clearly by the CNN.

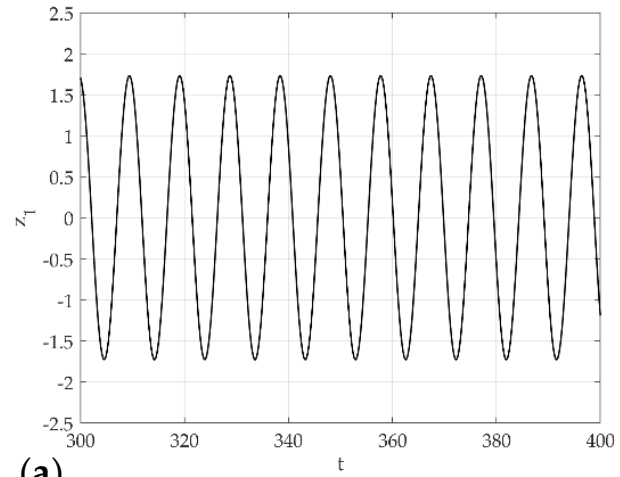

(a)

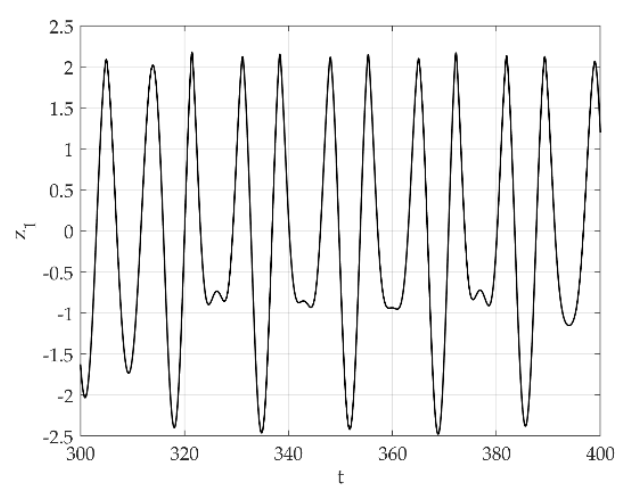

(c)
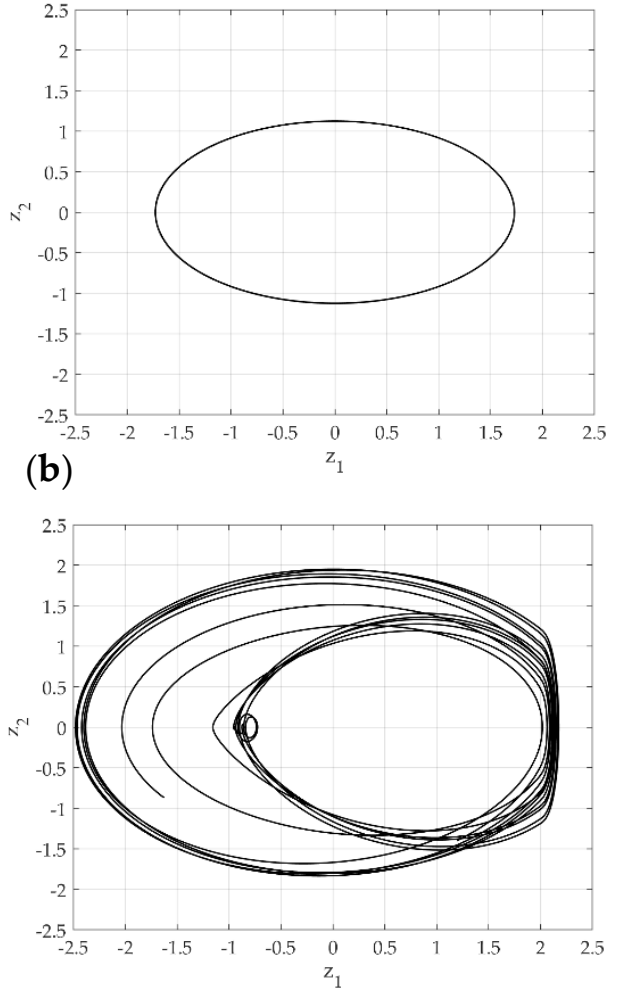

(d)

Figure 9. Trajectory, (a) time signal without impact, (b) phase portrait corresponding to (a), (c) time signal with impact, (d) phase portrait corresponding to (c).
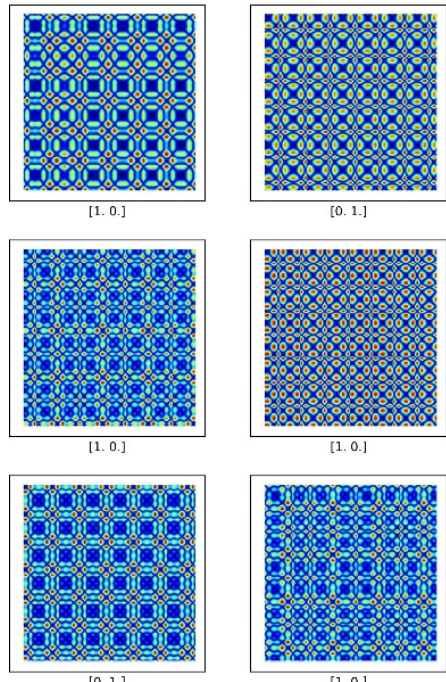

[1. 0.]
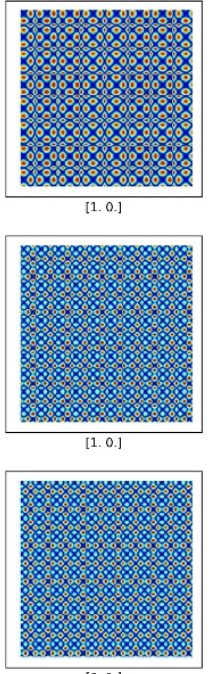

[0.11

Figure 10. Unthresholded recurrence plot by chaos and non-chaos (chaos: [1 0], non-chaos: [0 1]). 


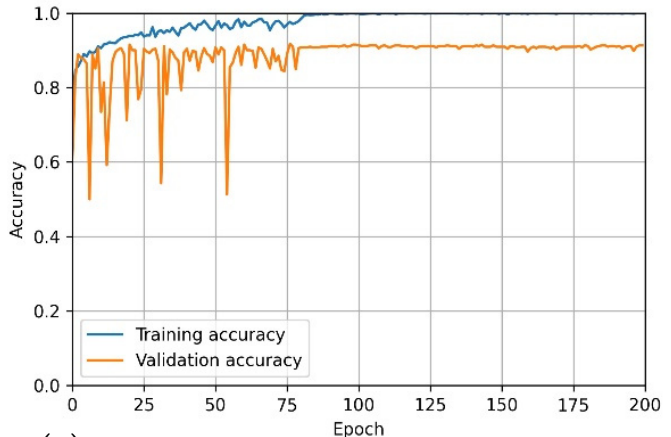

(a)

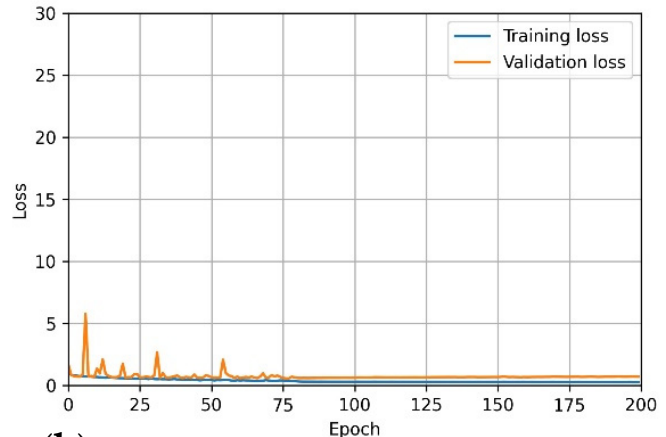

(b)

Figure 11. Unthresholded recurrence plot by (a) chaos and (b) non-chaos (chaos: [1 0], non-chaos: [0 1]).

\section{Discussions}

This study developed a CNN model that visualizes a dynamic signal with high order nonlinearity and distinguishes between chaos and non-chaos. In addition, the chaotic characteristics were verified by calculating the Lyapunov exponent. The signal generated in the dynamic system could be distinguished from the chaotic characteristics by calculating the Lyapunov exponent. On the other hand, the actual signals were complexly generated with noise and uncertainty. Visualization of the dynamic signals was used as a general method of analyzing the signal characteristics, but signals with nonlinearity are very difficult for engineers to analyze. In particular, chaotic signals cannot be classified accurately by the human cognitive ability, and theoretical analysis methods have limitations. CNN models have been developed that overcome the errors in human cognitive ability owing to the rapid development of artificial intelligence. Therefore, a simple CNN model that distinguishes between chaos and non-chaos with an accuracy of approximately $95 \%$ was developed. A new methodology that can analyze a real complex signal and more complex signals, such as BSR noise, will be discussed in the future.

In the future, this method of obtaining the feature learned from explicitly known equations and the Lyapunov exponent will be further applied to the experimental signal from which the Lyapunov exponent is not clearly calculated.

Author Contributions: Conceptualization, J.K.; methodology, J.K.; software, J.K.; validation, J.K. and J.N.; formal analysis, J.K. and J.N.; investigation, J.K. and J.N.; resources, J.K.; data curation, J.K.; writing, original draft preparation, J.K and J.N.; writing, review and editing, J.K. and J.N.; visualization, J.K. and J.N.; project administration, J.K.; funding acquisition, J.K. All authors have read and agreed to the published version of the manuscript.

Funding: This work was supported by Inha University Research Grant (INHA-63005-01).

Conflicts of Interest: The authors declare no conflict of interest.

\section{References}

1. Barrio, R.; Blesa, F.; Serrano, S. Qualitative analysis of the Rössler equations: Bifurcations of limit cycles and chaotic attractors. Phys. D 2009, 238, 1087-1100. [CrossRef]

2. Wei, D.; Zhu, W.; Wang, B.; Ma, Q.; Kang, Z. Effects of brake pressures on stick-slip bifurcation and chaos of the vehicle brake system. J. Vibroengineering 2015, 17, 2718-2732.

3. Oberst, S.; Lai, J. Chaos in brake squeal noise. J. Sound Vibrat. 2011, 330, 955-975. [CrossRef]

4. Li, Z.; Cao, Q.; Nie, Z. Stick-slip vibrations of a self-excited SD oscillator with Coulomb friction. Nonlinear Dyn. 2020, $102,1419-1435$. [CrossRef]

5. Wei, D.; Ruan, J.; Zhu, W.; Kang, Z. Properties of stability, bifurcation, and chaos of the tangential motion disk brake. J. Sound Vibrat. 2016, 375, 353-365. [CrossRef]

6. Wolf, A.; Swift, J.B.; Swinney, H.L.; Vastano, J.A. Determining Lyapunov exponents from a time series. Phys. D 1985, 16, $285-317$. [CrossRef]

7. Müller, P.C. Calculation of Lyapunov exponents for dynamic systems with discontinuities. Chaos Solitons Fractals 1995, 5, 1671-1681. [CrossRef] 
8. Kang, J.; Krousgrill, C.M.; Sadeghi, F. Comprehensive stability analysis of disc brake vibrations including gyroscopic, negative friction slope and mode-coupling mechanisms. J. Sound Vibrat. 2009, 324, 387-407. [CrossRef]

9. Kang, J. Numerical calculation of hip squeak over the normal gait cycle. Int. J. Precis. Eng. Manuf. 2019, 20, 2205-2214. [CrossRef]

10. Nam, J.; Do, H.; Kang, J. Investigation of friction induced vibration in lead screw system using FE model and its experimental validation. Appl. Acoust. 2017, 122, 98-106. [CrossRef]

11. Kang, J. Lyapunov exponent of friction-induced vibration under smooth friction curve. J. Mech. Sci. Technol. 2018, 32, 3563-3567. [CrossRef]

12. Serweta, W.; Okolewski, A.; Blazejczyk-Okolewska, B.; Czolczynski, K.; Kapitaniak, T. Lyapunov exponents of impact oscillators with Hertz's and Newton's contact models. Int. J. Mech. Sci. 2014, 89, 194-206. [CrossRef]

13. Serweta, W.; Okolewski, A.; Blazejczyk-Okolewska, B.; Czolczynski, K.; Kapitaniak, T. Mirror hysteresis and Lyapunov exponents of impact oscillator with symmetrical soft stops. Int. J. Mech. Sci. 2015, 101, 89-98. [CrossRef]

14. Kang, J. Calculation of Lyapunov exponents in impacted beam on distributed contact. J. Sound Vibrat. 2018, 431, 295-303. [CrossRef]

15. Hramov, A.E.; Koronovskii, A.A.; Makarov, V.A.; Pavlov, A.N.; Sitnikova, E. Wavelets in Neuroscience; Springer: Berlin/Heidelberg, Germany, 2015; pp. 45-72.

16. Goryunova, M.; Kuleshova, L.; Khakimova, A. Application of signal analysis for diagnostics. In Proceedings of the 2017 International Conference on Industrial Engineering, Applications and Manufacturing (ICIEAM), St. Petersburg, Russia, 16-19 May 2017; pp. 1-5.

17. Marwan, N.; Romano, M.C.; Thiel, M.; Kurths, J. Recurrence plots for the analysis of complex systems. Phys. Rep. 2007, 438, 237-329. [CrossRef]

18. Wallot, S.; Mønster, D. Calculation of average mutual information (AMI) and false-nearest neighbors (FNN) for the estimation of embedding parameters of multidimensional time series in matlab. Front. Psychol. 2018, 9, 1679. [CrossRef] [PubMed]

19. Adeli, H.; Ghosh-Dastidar, S.; Dadmehr, N. A wavelet-chaos methodology for analysis of EEGs and EEG subbands to detect seizure and epilepsy. IEEE Trans. Biomed. Eng. 2007, 54, 205-211. [CrossRef] [PubMed]

20. Zou, Y.; Donner, R.V.; Donges, J.F.; Marwan, N.; Kurths, J. Identifying complex periodic windows in continuous-time dynamical systems using recurrence-based methods. Chaos Interdiscip. J. Nonlinear Sci. 2010, 20, 043130. [CrossRef]

21. Butusov, D.N.; Karimov, A.I.; Pesterev, D.O.; Tutueva, A.V.; Okoli, G. Bifurcation and recurrent analysis of memristive circuits. In Proceedings of the 2018 IEEE Conference of Russian Young Researchers in Electrical and Electronic Engineering (EIConRus), Moscow, Russia, 29 January-1 February 2018; pp. 178-183.

22. Ozken, I.; Eroglu, D.; Stemler, T.; Marwan, N.; Bagci, G.B.; Kurths, J. Transformation-cost time-series method for analyzing irregularly sampled data. Phys. Rev. E 2015, 91, 062911. [CrossRef] [PubMed]

23. He, K.; Zhang, X.; Ren, S.; Sun, J. Deep residual learning for image recognition. In Proceedings of the IEEE Conference on Computer Vision and Pattern Recognition, Las Vegas, NV, USA, 27-30 June 2016; pp. 770-778.

24. Parker, T.S.; Chua, L. Practical Numerical Algorithms for Chaotic Systems; Springer Science \& Business Media: Berlin/Heidelberg, Germany, 2012.

25. Kingma, D.P.; Ba, J. Adam: A method for stochastic optimization. arXiv 2014, arXiv:1412.6980.

26. LeCun, Y.A.; Bottou, L.; Orr, G.B.; Müller, K. Efficient backprop. In Neural Networks: Tricks of the Trade; Springer: Berlin/Heidelberg, Germany, 2012; pp. 9-48.

27. Glorot, X.; Bengio, Y. Understanding the difficulty of training deep feedforward neural networks. In Proceedings of the Thirteenth International Conference on Artificial Intelligence and Statistics, Sardinia, Italy, 13-15 May 2010; pp. 249-256.

28. He, K.; Zhang, X.; Ren, S.; Sun, J. Delving deep into rectifiers: Surpassing human-level performance on imagenet classification. In Proceedings of the IEEE International Conference on Computer Vision, Santiago, Chile, 7-13 December 2015; pp. $1026-1034$. 